\title{
The Effect of Ordering Decisions by Choice-Set Size on Consumer Search
}

\author{
JONATHAN LEVAV \\ NICHOLAS REINHOLTZ \\ CLAIRE LIN
}

\begin{abstract}
Consumers frequently engage in sequential decisions. This article explores whether the order of these decisions can influence the manner in which consumers search through the possible choice options. Results from five studies suggest that ordering decisions by increasing (vs. decreasing) choice-set size leads to greater search depth (measured by both sampling count and decision time). Initial, smaller choice sets in increasing sequences appear to initiate a maximizing mind-set, which then persists even as participants encounter later, larger choice sets. These participants report a greater desire to maximize and are less satisfied with their decisions, consistent with research on chronic maximizers. In addition, they continue to exhibit maximizing behavior in subsequent, unrelated tasks, supporting a mind-set account of the differences in search. In sum, decision makers are proposed to be "sticky adapters": initial decision strategies seem to constrain the extent to which they adapt to new contexts.
\end{abstract}

\begin{abstract}
Watch out for the fellow who talks about putting things in order! Putting things in order always means getting other people under your control. (Denis Diderot)
\end{abstract}

$\mathrm{C}$ onsumers frequently engage in sequential decisions. For instance, grocery store shoppers fill their shopping baskets one item at a time, restaurant diners order their meal one course at a time, and computer buyers configure their laptops one component at a time. Each decision in the sequence might require choosing from a different number of options. Imagine that a homeowner who renovates her apartment engages in a sequence of decision steps: she might have to choose between 50 different tiles, 20 different wall-

Jonathan Levav (jlevav@stanford.edu) is associate professor of marketing at the Graduate School of Business, Stanford University, 655 Knight Way, Stanford, CA 94305. Nicholas Reinholtz (nsr2116@columbia.edu) is a doctoral student in marketing at the Columbia Business School, Columbia University, 3022 Broadway, New York, NY 10027. Claire Lin is a former doctoral candidate at the Columbia Business School who left academia to pursue a career as a thespian. The authors would like to thank Jim Bettman for his comments on an earlier draft of this article and the outstanding review team for their unusual dedication to improving this article.

John Deighton and Mary Frances Luce served as editors and Steve Hoch served as associate editor for this article.

Electronically published January 25, 2012 paint colors, and five different wallpaper designs. In this article, we explore how ordering decision sequences by choice-set size (i.e., ordering decisions according to their number of options) influences the depth of consumer search.

Order is important in many contexts, from impression formation (Asch 1946) to judgments of hedonic experiences (Ariely and Zauberman 2000). However, previous research about order in consumer search has limited its focus to the effect of ordering options within a specific choice set (e.g., Dellaert and Häubl 2012; Dellaert and Stremersch 2005; Diehl 2005; Diehl, Kornish, and Lynch 2003; Häubl, Dellaert, and Donkers 2010). The influence of decision order on search has, to our knowledge, remained unexplored.

Although there are many psychologically meaningful variables by which to order a decision sequence, we focus on order by choice-set size for two key reasons. First, set size can be objectively determined, and therefore it is an easy variable for firms to control. Second, and more importantly, previous research shows that set size can exert a substantial influence on consumers' psychology and consequently on their decisions. For example, large choice sets can demotivate purchases (Iyengar and Lepper 2000) or lead to decision simplification (Iyengar and Kamenica 2010). When people are given a large choice set, they are less likely to make a purchase than when they are given a small choice set. Further, choice-set size may affect decision confidence and preference strength (Chernev 2003). Finally, varying a configuration sequence by choice-set size can change peo- 
ple's ultimate product choice, even in high-stakes decisions such as automobile purchases (Levav et al. 2010).

Choice-set size can also influence the type of decision strategies that consumers adopt. It is well known that consumers are adaptive decision makers whose decision strategies are contingent on features of the decision environment (Payne 1976; Payne, Bettman, and Johnson 1993; Simonson and Tversky 1992). In particular, people's cognitive limitations lead them to seek ways to simplify their decisions. For instance, cognitive constraints can drive people to simplify decisions by adopting a satisficing goal (identifying a satisfactory option in a choice set), rather than a maximizing goal (identifying the best option in a choice set; Simon 1955, 1956), as a way to limit their decision effort and thus conserve their cognitive resources (Payne, Bettman, and Johnson 1988).

Maximizing and satisficing can manifest in people's search depth. For instance, in a study of college graduates, individuals who scored high on an 11-item maximization scale anticipated applying for twice as many jobs and actually attended more interviews than graduates who scored low on the same scale (Iyengar, Wells, and Schwartz 2006). In addition to such dispositional factors, environmental factors can also influence a person's likelihood to satisfice or maximize (Simon 1956). Large choice sets, for instance, appear to lead people to satisfice. Diehl (2005) reports that when people are offered a large choice set, they search a lower percentage of the set than when they are offered a small choice set. Similarly, Meyer (1997) finds that people truncate their search earlier when confronted with a large rather than small choice set. Thus, a small choice set appears to evoke exhaustive search, and a large choice set appears to evoke limited search.

Most of the prior experimental evidence regarding choiceset size and adaptive decision making has been limited to one-shot decisions. However, in many purchase situations (e.g., configuration decisions), consumers make a sequence of multiple decisions, rather than a single decision on its own. Suppose our homeowner from the example above begins the sequential process of renovation decisions by choosing from a small set of five wallpaper designs. Previous research suggests that she would search a high proportion of the alternatives (Diehl 2005), presumably with a greater desire to maximize her choice outcome. What happens when she moves to a subsequent decision in which she must choose from a larger set of options (e.g., 20 wall-paint colors)? Will she adapt to the larger choice set by altering her search strategies to cope with the new decision environment and adopt a satisficing heuristic?

Research on problem solving suggests that our homeowner might adapt her initial strategy based on the requirements of the first decision problem but persist in using the same strategy in subsequent decisions regardless of its suitability. Classic demonstrations of the "Einstellung effect" in problem solving indicate that once people learn a rule for successfully solving an initial series of problems, they rigidly apply this rule to later problems as well, even when easier rules are obvious. In Luchins's (1942) water-jug experiment, participants were asked to measure out a given amount of water using a combination of three jugs of various capacities. The practice trials consisted of problems that required a relatively complicated solution. In the target problem, even though there was a simpler solution that was easily detectable by control participants who had not engaged in practice trials, participants in the treatment group persisted in using the complicated solution. Similarly, in the consumer domain, studies show that context-driven decision rules can persist even after the initial context is removed (Amir and Levav 2008; Häubl and Murray 2003). Broder and Schiffer's (2006) studies on sequential stock-market decisions show that once people adopt a decision strategy, they tend to persist with that strategy even when the decision environment changes and the strategy yields economically suboptimal outcomes. The tendency to retain response patterns, even when they cease to be beneficial, has been termed "behavioral stereotypy" (Schwartz 1982). In sum, prior research suggests that the initial search strategy used by a consumer may be "sticky," persisting in later decisions.

A possible explanation for the persistence of a decision strategy comes from work on consumer mind-sets. Mindsets have been defined broadly in consumer research: they can refer to the cognitive or motor procedures (Wyer and Xu 2010), judgmental criteria (Xu and Wyer 2007), or goals (Keinan and Kivetz 2011) that are triggered by a task and subsequently generalized to different tasks or contexts. For instance, $\mathrm{Xu}$ and Wyer (2007) suggest that considering a purchase in category $x$ increases the probability of purchase in a subsequent, unrelated category $y$ because the act of consideration places consumers in a "which-to-buy" mindset. Similarly, Ülkümen, Chakravarti, and Morwitz (2010) report that an initial task that uses broader rather than narrower categories leads people to create broader rather than finer grouping of items in a subsequent, unrelated task. In this article, we follow Keinan and Kivetz's (2011) conceptualization of mind-set as the persistence of goals and motivations.

We suggest that the initial decision in a sequential choice task evokes a mind-set-maximizing versus satisficing-in the consumer. As a result, we hypothesize that the strategy used for the first choice in a sequence will carry forward and influence the strategy used in subsequent choices, even though these choices will be from choice sets of different sizes. In our homeowner example, this implies that if she begins her renovation decision process with a small choice set (i.e., wallpaper, five options), she will search this set more exhaustively and initiate more of a maximizing mindset than if she had started with a larger choice set (i.e., tiles, 50 options). As our homeowner proceeds to choices with a greater number of options (i.e., wall-paint colors, 20 options), her relative tendency to maximize will lead her to search more exhaustively than if she had started with the larger set. The cumulative effect of this strategy stickiness is that she will ultimately search more options when she begins with a small choice set and proceeds to larger choice 
sets than if she begins with a large choice set and proceeds to smaller choice sets. If she were completely adaptive in her decision strategies, choice-set order would not matter to how she searched or what she chose.

\section{OVERVIEW}

Our empirical section reports the results of five studies. In experiment 1 , using an experience good (music), we test the basic hypothesis that ordering sequential decisions by increasing or decreasing choice-set size leads to differences in search depth. We show that participants who encounter choice sets with an increasing number of options sample a larger proportion of the total options than those who encounter choice sets with a decreasing number of options. In experiment 2, we generalize this effect to search goods (household products) that uses a measure of search depth inspired by research using information boards. In experiment 3 , we examine the mechanism behind our findings in experiments 1 and 2 by explicitly measuring the participants' search goals - the extent to which they report maximizing or satisficing. We also examine the downstream effect of our sequence manipulation on satisfaction. In experiments 4 and 5, we present further evidence supporting a mind-set account of decision strategy persistence. Experiment 4 shows that the sequence effect persists even when participants switch to an unexpected "bonus" task in the same domain. Experiment 5 provides evidence that the relative tendency to maximize initiated by sequence order can also carry over to a task in a completely different domain.

\section{EXPERIMENT 1}

Experiment 1 tests the hypothesis that decision sequence affects search depth. In this experiment, all participants select one item from each of 10 choice sets. All participants encounter the same choice set sizes; however, we manipulate whether participants encounter these choice sets from smallest to biggest (increasing) or from biggest to smallest (decreasing). Because we hypothesize that small choice sets will lead to a more exhaustive search and a "sticky" tendency to maximize choice, we predict that participants who encounter the choice sets in an increasing order will sample a greater number of options and spend more time searching than their counterparts who encounter the same-sized choice sets in a decreasing order.

\section{Method}

Participants. Forty-nine undergraduate and graduate students participated in experiment 1 , which we advertised as a "marketing study of MP3 songs." Participants received \$8 and a customized music CD as compensation.

Design and Procedure. Participants were told that, as part of a marketing study, they were being asked to create a CD with 10 tracks (songs) using a computer-based customization interface. They were told to imagine that they were taking a road trip and that the CD they were about to create should contain songs they would like to listen to on that trip. In order to make participants' choices consequential, we informed them that they would actually receive the CD that they configured. The options for each track were randomly selected from a pool of 2,327 pop-music songs by unfamiliar artists.

Participants proceeded through a series of 10 song-selection screens, each corresponding to a track on the participant's CD. On each screen, they were presented with a list of songs (displayed by name) and asked to choose one for their CD. Participants could listen to a song by clicking on its name. The program was self-paced, and participants could sample as many songs as they wished. They were also free to interrupt a song or listen to it again. Once participants chose a song for a given track, they continued to the next track and could not go back to change their decision.

The number of options for each track varied depending on the experimental condition. Participants in the increasing condition encountered an increasing number of options from track 1 to track 10 in increments of five (five options for track 1, 10 options for track 2 . . . and 50 options for track 10). In contrast, participants in the decreasing condition encountered a decreasing number of options from track 1 to track 10 in decrements of five (50 options for track 1, 45 options for track 2 . . . and five options for track 10). Thus, we employed a 2 (sequence: increasing vs. decreasing) $\times$ 10 (choice-set size: 5,10 . . . 50) mixed design with sequence as a between-participants factor and choice-set size as a within-participant factor. Figure 1 graphically represents our experimental paradigm.

The software recorded two measures that reflect search depth: (1) the number of songs that a participant sampled (sampling count) and (2) the amount of time the participant spent choosing a song for each of the 10 tracks (decision time).

\section{FIGURE 1}

\section{PARADIGM FOR EXPERIMENT 1: ORDERING SEQUENTIAL PRODUCT DECISIONS BY INCREASING VERSUS DECREASING CHOICE-SET SIZE}

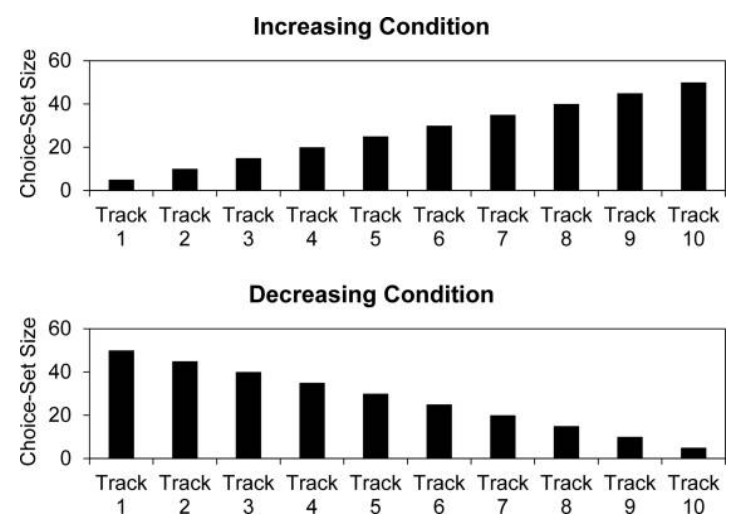




\section{Results}

The results supported our hypothesis that participants' depth of search would be sensitive to the sequence manipulation. This is evident in both their sampling count and decision time.

Sampling Count. An ANOVA with sequence as a between-participants factor and choice-set size as a withinparticipant factor indicated a significant main effect of choice-set size $(F(9,423)=12.99, p<.01)$. The top panel of figure 2 shows that participants in both conditions generally sampled more songs from the larger choice sets. As suggested by previous research, this tendency was not monotonic (Shah and Wolford 2007). More importantly, our sequence manipulation had a significant main effect on sampling count: participants in the increasing condition sampled an average of 149 unique songs (out of a possible 275), compared to only 101 songs by participants in the decreasing condition $(F(1,47)=8.73, p<.01)$. Thus, even though all participants had encountered the same total number of options, those in the increasing condition searched almost 50\% more than those in the decreasing condition.

We also compared participants' search depth for each track, while holding the number of songs constant (e.g., the five-option choice set for track 1 in the increasing condition is compared with the five-option choice set for track 10 in the decreasing condition; see fig. 2). Planned contrasts revealed that participants in the increasing condition sampled significantly more songs in eight out of the 10 tracks (all $F$ $>3.4$, all $p<.05$ ). The exceptions were the smallest (five options; $M_{\text {inc. }}=4.68$ vs. $M_{\text {dec. }}=4.96 ; F(1,47)=1.38, p$ $=.25$ ) and largest (50 options; $M_{\text {inc. }}=12.56$ vs. $M_{\text {dec. }}=$ $14.96 ; F(1,47)=.45, p=.5)$ choice sets. We speculate that a ceiling effect accounts for the result in the five-option set-with such a limited number options, participants in both groups tended to be thorough despite the manipulation. Furthermore, we speculate that in the 50-option set, participants in the decreasing condition searched more than expected, perhaps because they had just begun the experiment, and participants in the increasing condition searched less than expected, perhaps because they anticipated the experiment's completion. Note, however, that this pattern does not emerge consistently across our studies and that our focus in this article is on the overall search amount throughout the entire decision sequence.

Percentage Searched. The previous analysis suggests that a participant's search strategy is sticky throughout the decision sequence. However, participants did show adaptivity in terms of the percentage of each choice set that they searched. Thus, they were not completely insensitive to choice-set sizes: on average, participants searched over $90 \%$ of the options in the smallest choice set but searched less than half of the options in the largest choice set. We plot the data in terms of percentage searched in the bottom panel of figure 2. While this mode of presentation makes it easier to discern adaptivity among our participants, it is still evident that our sequence manipulation influenced people's search
FIGURE 2

\section{EXPERIMENT 1: SEARCH DEPTH BY CHOICE-SET SIZE}
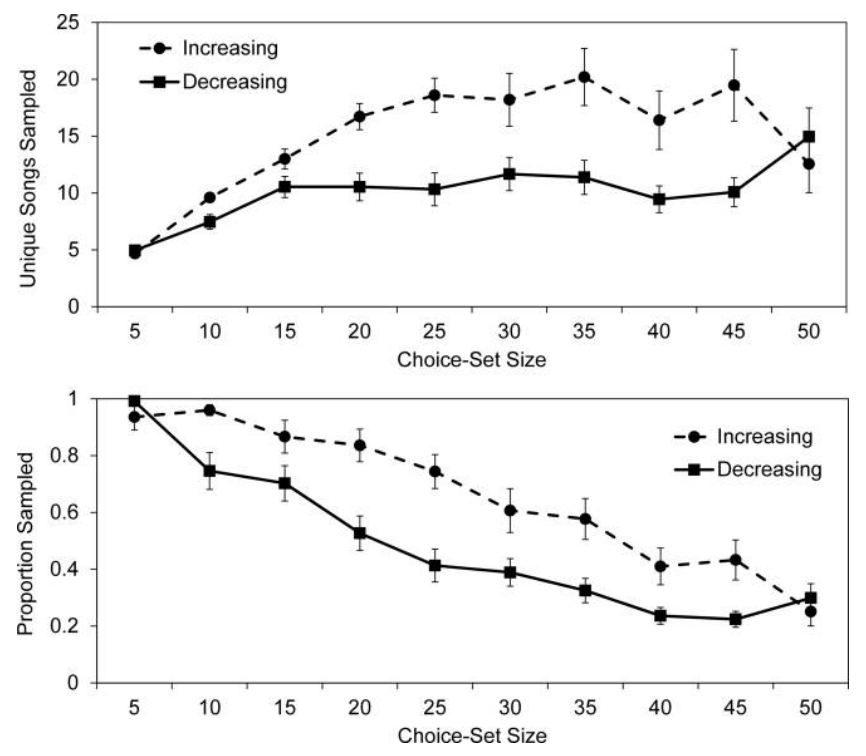

depth. A reanalysis of the data using percentage searched as the dependent variable yields two significant main effects, as before: participants in both conditions searched a greater percentage from smaller choice sets $(F(9,423)=73.2, p$ $<.01$ ), showing evidence of adaptivity, but participants in the increasing condition searched a greater percentage on average than their decreasing condition counterparts $(F(1$, $47)=10.02, p<.01)$, showing evidence of stickiness.

While the qualitative insights gained from analyzing percent searched are similar to the insights gained from analyzing sampling count, here we believe it is useful to present both because the percent-searched presentation helps illustrate the fact that the strategy stickiness we propose is not absolute. For the sake of brevity, in subsequent studies, we report only the analysis based on sampling count because we believe it is a more direct measure of the effort expended during search. However, note that performing the same analyses using percent searched (when possible) in the studies that follow yields similar results and qualitative conclusions.

Decision Time. The results for decision time closely parallel those of sampling count. Participants in the increasing condition spent more time overall than their decreasing condition counterparts $\left(M_{\text {inc. }}=2,412\right.$ seconds vs. $M_{\text {dec. }}=1,566$ seconds; $F(1,47)=2.9, p<.01)$. However, note that participants in the increasing condition listened to more songs; thus, the amount of time spent per song was approximately the same in both conditions $\left(M_{\text {inc. }}=16.8\right.$ seconds vs. $M_{\text {dec. }}$. $=16.3$ seconds; $F(1,47)=.23, p=.56)$. As an additional indicator of search depth, we analyzed the amount of time participants spent searching after they listened to the song that they eventually chose. In other words, we examined the 
time participants spent between sampling the song that they eventually chose and making it their final selection. We found that participants in the increasing condition searched significantly longer in this period than those in the decreasing condition $\left(M_{\text {inc. }}=1,387\right.$ seconds vs. $M_{\text {dec. }}=796 \mathrm{sec}-$ onds; $F(1,47)=9.9, p<.01$ ). (We replicate this result in our subsequent studies.)

\section{Discussion}

In this experiment, we find that decision order can influence the depth of consumers' search. In particular, ordering decisions by increasing set size fosters deeper search, in terms of both total number of items sampled and the total length of time spent searching. Note that participants in this study essentially made the same kind of decision 10 times. We chose this paradigm in order to isolate the effect of sequence without the potentially confounding factor of different product categories. However, in many sequential decision contexts, consumers make choices from different categories, as in our opening example of home renovation. In the next study, we test whether our effect generalizes to such contexts.

\section{EXPERIMENT 2}

In experiment 2, participants simulated a shopping trip in which they made a sequence of eight decisions, each involving a choice in a different product category. We created an online store in which participants were offered an opportunity to choose from eight common household product categories in succession. A key difference between this study and experiment 1 is that participants could not actually experience the products. Instead, they saw thumbnail photos of each option and could mouse-over the thumbnail in order to see an enlarged photo, the product's price, and a brief description of the product. A second difference is that by providing price, we gave participants an alignable attribute on which to base their search; previous research shows that people display different search patterns for items with alignable (vs. nonalignable) attributes (Griffin and Broniarczyk 2010). We measured participants' search depth by recording their frequency of mouse-overs, as is common in processtracing studies such as those conducted using Mouselab (Johnson et al. 1988).

\section{Method}

Participants. One hundred and sixty-eight undergraduate and graduate students participated in this experiment in exchange for $\$ 10$.

Design and Procedure. Participants were told that the study was about people's online shopping behavior. They were asked to shop within eight product categories that were presented sequentially in a computer-simulated, online shopping environment. To ensure that participants would treat the task as consequential, they were asked to pay for two of the items that they selected during the session (randomly selected from their eight choices):

In this study, you will be making choices from eight product categories in an electronic store. Like any other store, each product will have a price. To compensate you for your participation, you will receive $\$ 10$ in cash. At the end of the study, two of the product categories will be chosen at random. You will pay for and receive the items that you selected for these two categories. The difference between your $\$ 10$ cash allowance and the total price of the two products is yours to keep (in addition to the products). So, for example, if you chose an apple that cost $\$ 1$ and a chocolate bar that costs $\$ 1.50$, you will receive $\$ 10.00-\$ 2.50$, or $\$ 7.50$, and you will also receive the apple and the chocolate bar. In other words, each decision you make should be treated like an actual purchase-so buy what you would like to take home and are willing to pay for!

After the introduction, participants proceeded with the shopping task. We selected eight common product categories featuring items priced around or below $\$ 5$ : body lotions, cereal, energy bars, jams, notebooks, pens, shampoo, and temporary tattoos. For each product category, we identified a pool of 50 options from actual online stores and randomly drew the stimuli for each participant from these pools of options.

Each of the eight product categories appeared on a separate screen, with thumbnail images for each product displayed in a matrix. In order to view a product's price and description, participants had to hover the mouse pointer over the product's thumbnail image. After selecting their preferred product in a category using a mouse click, participants proceeded to the next screen. The participant's (virtual) shopping cart appeared on the right-hand side of the screen and displayed a list of items in the cart and their running total price. They could not revise their previous choices.

The number of options for each product category varied depending on the experimental condition. Participants in the increasing condition encountered an increasing number of options as they proceeded from category to category (i.e., choice-set size: $5,8,13,17,20,23,26,30)$, while participants in the decreasing condition encountered the reverse order. To control for possible category order effects, we counterbalanced the product categories such that participants shopped through the product categories in either alphabetical order (i.e., from body lotions to temporary tattoos) or reverse-alphabetical order (i.e., from temporary tattoos to body lotions). For example, participants in the increasingalphabetical condition were presented with the following shopping sequence: body lotions (five options), cereal (eight options) . . . temporary tattoos (30 options). In contrast, participants in the increasing-reverse-alphabetical condition were presented with the following shopping sequence: temporary tattoos (five options), shampoos (eight options) . . . body lotions (30 options). Thus, we employed a 2 (sequence: increasing vs. decreasing) $\times 2$ (product order: alphabetical 
vs. reverse-alphabetical) $\times 8$ (choice-set size: $5,8,13,17$, 20, 23, 26, 30) mixed design.

The software recorded the number of mouse-overs by each participant in each category. After participants completed the task, they were debriefed and informed that they would actually receive the entire $\$ 10$ compensation as well as two of the items that they chose for free.

\section{Results}

The results of this experiment replicate those of experiment 1. Following previous research using the Mouselab software to track search patterns, we used information acquisition-the number of times people mouse-over a product thumbnail to acquire information about the product-to measure people's search depth (e.g., Dhar, Nowlis, and Sherman 2000; Goukens, DeWitte, and Warlop 2009; Payne et al. 1988). However, since mousing-over required minimal time and effort compared with sampling music in experiment 1 , we expected and found a ceiling effect-almost every participant moused-over each item at least once. Thus, we used a more sensitive measure of search effort in which we normalized information acquisition by individual consideration-set size (defined in our case as the total number of products in a category that the participant moused-over). That is, we calculated the average number of times participants examined (i.e., moused-over) each option in their consideration set.

We conducted an ANOVA with our normalized information acquisition measure as the dependent variable and set size, sequence, and product order as independent variables. The results indicated that neither the effect of product order $(F(1,164)=.52, p=.47)$ nor the interaction of product order by sequence $(F(1,164)=.69, p=.41)$ was significant. The effect of choice-set size, the within-participant factor, on information acquisition was highly significant $(F(7,1148)=52.24, p<.001)$, indicating that, regardless of condition, people tended to search more thoroughly in smaller choice sets. This was qualified by a significant interaction between the sequence manipulation and choice-set size $(F(7,1148)=11.69, p<.001)$, suggesting the pattern of search within the choice sets was different between conditions. Noticeably, participants in the increasing condition searched less in the last few sets, which could perhaps be indicative of fatigue. More importantly, the sequence manipulation was a significant predictor of information acquisition $\left(M_{\text {inc. }}=2.36, M_{\text {dec. }}=2.15 ; F(1,164)=5.41, p=\right.$ $.02)$. That is, on average, participants in the increasing condition looked up the description of each option that they were considering more times than participants in the decreasing condition (see fig. 3). The difference in the level of search between conditions did not translate into a difference in the total price of the chosen items $\left(M_{\text {inc. }}=\$ 37.02\right.$, $\left.M_{\text {dec. }}=\$ 36.63 ; t(166)=.46, p=.64\right)$.

\section{Discussion}

Using a different operationalization of search depth, we replicate the results of experiment 1 by showing deeper
FIGURE 3

\section{EXPERIMENT 2: INFORMATION ACQUISITION BY CHOICE-SET SIZE}

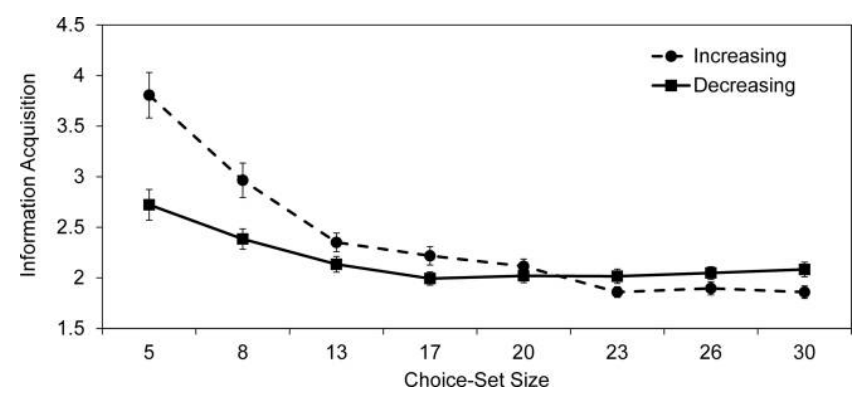

search in sequences ordered by increasing set size. A potential alternative explanation for the results of this and the previous experiment is that participants' search was influenced by the expectations they formed about the task as a result of the first choice set. For instance, participants in the increasing conditions might have incorrectly anticipated having a sequence of mostly small choice sets to evaluate. Similarly, participants in the decreasing conditions might have incorrectly anticipated mostly large choice sets in subsequent decisions. This difference in expectations might have led participants in the increasing conditions to believe that they could afford to spend more time on each decision and participants in the decreasing conditions to believe that they could not afford to do so. We address this alternative explanation about set-size expectations in the next experiment.

\section{EXPERIMENT 3}

We had four objectives in experiment 3. The first was to address the alternative explanation about set-size expectations. To this end, we added a factor to the experiment in which participants were either told explicitly the order and size of the choice sets that they would encounter or, as in our previous studies, not told these details. Second, in order to assess whether our manipulation influenced the participants' mind-set (maximizing vs. satisficing), we explicitly measured participants' search goals. Third, because past research on chronic maximizers suggests that greater search leads to lower satisfaction with decision outcomes (Iyengar et al. 2006), we used a behavioral measure of satisfaction to test whether participants in the increasing condition would also be less satisfied with their choices. Finally, we added a condition in which all set sizes were approximately equal in order to assess the effect of our manipulation relative to a baseline. 


\section{Method}

Participants. One hundred and five undergraduate and graduate students participated in the study in exchange for $\$ 8$ and the opportunity to download 10 songs.

Design and Procedure. We employed a 2 (sequence: increasing vs. decreasing) $\times 2$ (information: informed vs. uninformed) $\times 10$ (choice-set size: 5,10 . . 50) mixed design. Sequence and information were between-participants factors, while choice-set size was a within-participant factor. In addition, we included a baseline condition in which participants made 10 decisions, all of which had a similar number of options (in order to equal 275 options as in the other conditions, half of the decisions were randomly assigned to have 27 options, and the other half were randomly assigned to have 28 options). Participants in the baseline condition were not provided any information about the sequence.

The procedure was the same as in experiment 1, using the same stimuli, with four important exceptions. First, after reading the same instructions used in experiment 1 , but prior to beginning the task, participants in the informed conditions were told exactly what their decision sequence would be. Specifically, participants in the informed-increasing condition were told that they would choose between five options for the first track of the CD, 10 options for the second track of the CD, and so on. Participants in the informed-decreasing condition were told that they would have 50 options for the first track of the CD, 45 options for the second track of the $\mathrm{CD}$, and so on. Second, at the end of the experiment, participants were asked to estimate the total number of options they had been offered. We used this measure as a manipulation check to make sure that participants in the informed condition had indeed paid attention to the information about the sequence. Third, following the estimation task and an additional filler task, all participants completed a short questionnaire to assess the extent to which they were employing a maximization strategy using items adapted from an established maximization scale (Schwartz et al. 2002; see appendix A). Finally, in lieu of downloading their selected music immediately, participants were told that they would receive an e-mail providing them with a link to the music 2 weeks from the date of the experiment. We used participants' download activity as a proxy for their downstream satisfaction with their choices.

\section{Results}

The results of this experiment cast doubt on an alternative explanation based on set-size expectations and confirm that our manipulation influenced participants' self-described search goals.

Manipulation Check. The information manipulation was effective. Although the average estimated number of songs in both conditions was significantly different from the correct number, $275\left(t_{\text {inf. }}(41)=-2.48, p=.01 ; t_{\text {uninf. }}(41)=\right.$ $-9.20, p<.001)$, participants in the informed conditions estimated the number of songs more accurately than those who had not been informed $\left(M_{\text {inf. }}=238\right.$ vs. $M_{\text {uninf. }}=154$; $F(1,82)=17.59, p<.001)$.

Search Depth and Information. We conducted a mixed ANOVA with sampling count as the dependent variable and sequence, information, and choice-set size as independent variables (we analyze the baseline condition separately, below). Replicating our previous experiments, we find a significant main effect of sequence on search depth: participants in the increasing condition sampled more songs than those in the decreasing condition $\left(M_{\text {inc. }}=164\right.$ vs. $M_{\text {dec. }}=127$; $F(1,80)=4.85, p=.03)$. Importantly, information did not have a main effect on search depth $(F(1,80)=.60, p$ $=.44)$ and did not interact with sequence $(F(1,80)=.26$, $p=.61)$. See figure 4 . We also found a significant main effect of choice-set size $(F(9,720)=3.46, p<.001)$ and its interaction with condition $(F(9,720)=35.06, p<.001)$, which indicate different average levels of search in the different-sized choice sets. The analysis of decision time revealed an identical pattern. In other words, informing participants in advance about the exact composition of the sequence did not influence their search behavior or eliminate the effect of the sequence manipulation. This result casts doubt on the alternative explanation that the influence of the sequence manipulation is due to false set-size expectations.

Baseline Condition. We compared the total sampling count in the increasing and decreasing conditions with the total sampling count in the baseline condition, collapsing across the information factor. Sampling count in the in-

\section{FIGURE 4}

EXPERIMENT 3: SAMPLING COUNT (INFORMED VS. UNINFORMED)
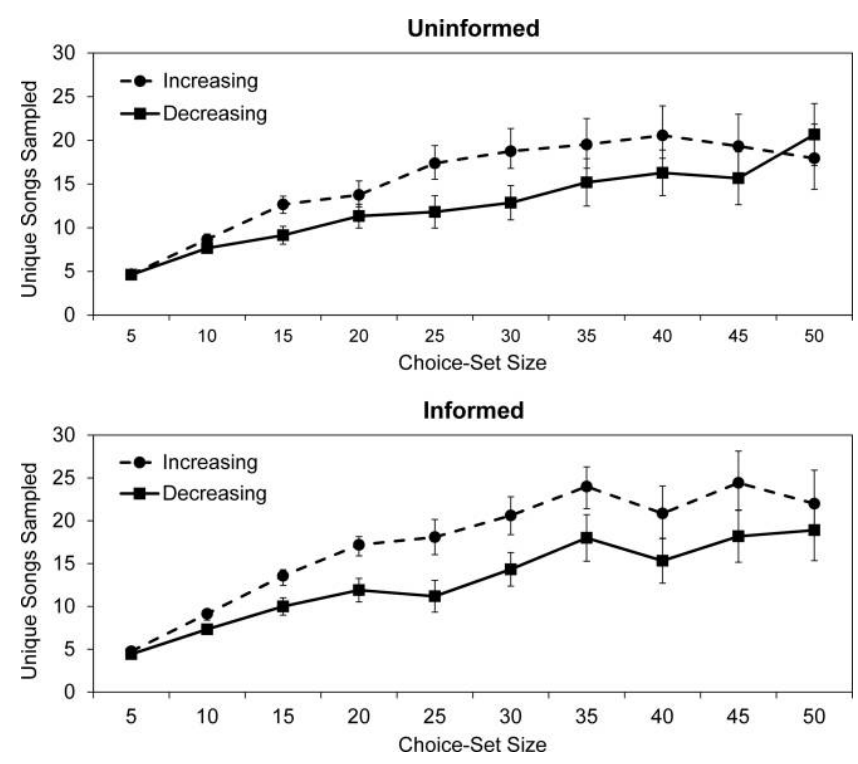
creasing condition was significantly higher than sampling count in the baseline condition $\left(M_{\text {inc. }}=164\right.$ vs. $M_{\text {base }}=$ $98 ; F(1,102)=12.37, p<.001)$, but there was no significant difference between the decreasing condition and the baseline condition $\left(M_{\text {dec. }}=127\right.$ vs. $M_{\text {base }}=98 ; F(1,102)$ $=2.47, p>.10$ ). Thus, it appears that the difference in depth of search that we find is driven by greater search in the increasing condition, which suggests that our manipulation is effective at inducing a maximizing mind-set. This result is also consistent with Simon's (1955) notion that people are naturally prone to satisficing-our research suggests a simple manipulation that prompts people to overcome this default tendency.

Mediation Analysis. Next we tested whether the observed search depth differences were mediated by the decision mind-set of the participants. To this end, we averaged participants' responses to the four questions, measuring their search goals to form a maximization mind-set index (Cronbach's $\alpha=.69$ ). A high average indicates a greater tendency to maximize, and a low average indicates a greater tendency to satisfice. Thus, we treat these two decision strategiesmaximizing and satisficing - as opposite poles of a continuous dimension. This conceptualization is consistent with Schwartz et al. (2002), who acknowledge that "it is surely more accurate to say that people differ in the extent to which they are maximizers, rather than falling on one side or the other of the maximization line" (1194).

Participants in the increasing condition reported higher levels of maximization tendency than their counterparts in the decreasing condition $\left(M_{\text {inc. }}=1.46, \mathrm{SD}_{\text {inc. }}=.75 \mathrm{vs} . M_{\text {dec. }}\right.$. $\left.=.95, \mathrm{SD}_{\text {dec. }}=1.25 ; F(1,82)=5.06, p=.03\right)$. To test for mediation, we used the Preacher and Hayes (2004) method for estimating indirect effects. Consistent with our hypothesis, bootstrap estimates (based on 5,000 samples) indicated that the indirect effect of sequence (increasing vs. decreasing) through the maximization-mind-set index had a significant effect on depth of search (point estimate: 17.4, 95\% confidence interval: 2.6-34.4). When maximization mind-set was included in the regression, the direct effect of sequence on search depth became nonsignificant $(\beta=$ $19.16, t=1.27, p=.21)$. According to Zhao, Lynch, and Chen (2010), this pattern of results suggests indirect-only mediation.

While the mediation results are consistent with the hypothesis that choices ordered by increasing set size initiate a maximization mind-set in the decision maker, an alternative view of this mediation analysis reverses the causality implied in our interpretation. In particular, consistent with self-perception theory (Bem 1972), participants might have inferred their mind-sets by thinking back to their search behavior and assessing its relative depth. Thus, a conservative interpretation of our finding is simply that our manipulation affects both search behavior and (measured) maximization tendency.

Satisfaction. To test whether people in the increasing condition show the same tendency for decision dissatisfac- tion as chronic maximizers, we analyzed download activity as a behavioral measure of satisfaction. In the increasing condition, only $41 \%$ of participants downloaded their songs, compared with $62 \%$ in the decreasing condition. A logistic regression with sequence and information as predictors indicated that this difference in proportions is significant $\left(\chi^{2}\right.$ $=3.8, p=.05)$, suggesting that the increasing condition led to greater search but lower satisfaction, just as expected of chronic maximizers. Note that our analysis revealed neither a main effect of information $\left(\chi^{2}=.05, p=.83\right)$ nor an interaction between information and sequence $\left(\chi^{2}=.05\right.$, $p=.83)$. Thus, it appears that the information manipulation did not have a downstream effect on participants' satisfaction.

\section{Discussion}

Across three studies, we replicate our effect: a sequence of increasing choice-set sizes triggers deeper search than a sequence of decreasing choice-set sizes. Experiment 3 suggests that this effect is largely driven by greater search in increasing sequences. Furthermore, our effect does not appear to be an artifact of participants' set-size expectations. This experiment also sheds light on the mechanism behind the observed effect: A mediation analysis suggests that the observed differences in search depth could be driven by changes in the participants' decision-strategy mind-set (i.e., the extent to which participants are maximizing vs. satisficing). Finally, consistent with past research about chronic maximizers, we find that participants in the increasing condition search more but are less satisfied with their eventual choices.

\section{EXPERIMENT 4}

An important feature of mind-sets as we have used them here is that they carry over from task to task. If the manipulation of decision order evokes a certain mind-set, then we should observe this characteristic carryover in our choice tasks as well. To this end, in this experiment, we add an additional task at the end of the decision sequence that is identical across conditions. If participants in the increasing condition are indeed adopting a maximizing mind-set, we should expect them to search more deeply than their decreasing condition counterparts in this last task. A second feature of experiment 4 is that we reduce the number of decisions participants have to make in order to test whether search depth differences are also evident in shorter decision sequences.

\section{Method}

Participants. Fifty-nine graduate and undergraduate students participated in this experiment in exchange for $\$ 5$ and the opportunity to download four songs.

Design and Procedure. Participants were told that they would be presented with three music collections and that 
they would be asked to choose a song from each collection. We used the same stimuli as in experiment 1. Participants were randomly assigned to one of two conditions: in the increasing condition, the three music collections were ordered by increasing set size (i.e., five songs, 20 songs, and 50 songs), and in the decreasing condition the three music collections were ordered by decreasing set size (i.e., 50 songs, 20 songs, and five songs).

After participants completed their choices from the three music collections, they were presented with a surprise "bonus" 50-song music collection. The songs in this collection were the same for all participants, although their display order on the screen was randomized. Participants were asked to choose an additional song from this bonus music collection. We recorded the number of songs that they sampled and their total decision time.

\section{Results}

For the initial sequence of three choices, we replicated our previous results. An analysis of sampling count revealed that participants in the increasing condition sampled more songs than their decreasing condition counterparts $\left(M_{\text {inc. }}=\right.$ 36.6 songs vs. $M_{\text {dec. }}=25.7$ songs; $F(1,57)=5.13, p=$ $.03)$. This is illustrated in figure 5 . We obtained similar results for decision time $\left(M_{\text {inc. }}=574\right.$ seconds vs. $M_{\text {dec. }}=$ 406 seconds; $F(1,57)=6.22, p=.02)$.

More importantly, the effect of sequence on search depth carried over to the bonus task. Participants in the increasing condition sampled more options $\left(M_{\text {inc. }}=20.4\right.$ songs vs. $M_{\text {dec. }}$ $=13.4$ songs; $F(1,57)=3.45, p=.067)$ and searched longer $\left(M_{\text {inc. }}=230\right.$ seconds vs. $M_{\text {dec. }}=145$ seconds; $F(1,57)=$ $6.22, p=.02$ ) than those in the decreasing condition. Similar to experiments 1 and 3, across all choices, we find that participants in the increasing condition continued to search longer after sampling the song they eventually chose $\left(M_{\text {inc. }}\right.$ $=438$ seconds vs. $M_{\text {dec. }}=302$ seconds; $F(1,57)=4.57$, $p=.037)$. These results are supportive of a mind-set account of the observed difference in search depth.

\section{Discussion}

Experiment 4 has three implications. First, we find that our manipulation is robust to changes in sequence length. Second, the carryover effect that we find in the bonus task offers evidence consistent with a mind-set account of our sequence effect-namely, that the tendency to maximize initiated by the sequence manipulation can carry over to a subsequent, unexpected search task. Note that all participants had been exposed to the same number of songs (grouped in the same-sized sets) by the time they reached the bonus task. This fact helps rule out an alternative explanation based on resource depletion (Baumeister et al. 1998), which posits that participants in the decreasing conditions expend more cognitive energy on the first choice set(s) and thus become more cognitively fatigued, leading them to search less in the later choice sets. Our data are inconsistent with this alternative. Prior to the bonus task, participants in the de-

\section{FIGURE 5}

EXPERIMENT 4: SEARCH DEPTH (SAMPLING COUNT)

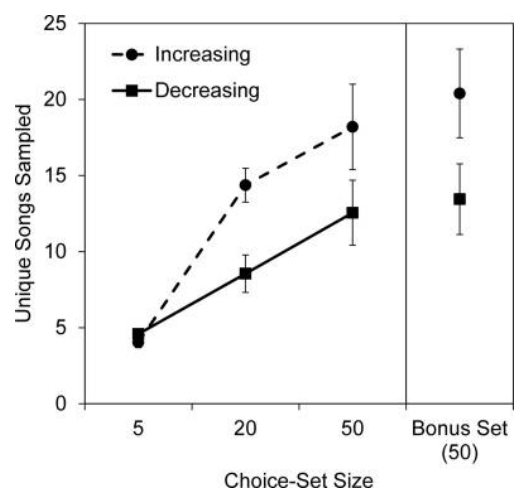

creasing condition had searched fewer options and had taken less time than their counterparts in the increasing condition. One would thus expect participants in the increasing condition to be more depleted, yet we find that the participants in the increasing condition continue to expend more energy, searching longer and more deeply in the bonus task.

\section{EXPERIMENT 5}

In experiment 4 , we find evidence for carryover in a task within the same domain as the initial decision sequence. However, activation of a mind-set should also influence subsequent tasks in unrelated domains. We test this prediction in the present study. Further, unlike the previous study, in which the values of the choice options were subjective, in this experiment, we test carryover in a domain with an objective outcome measure. Finally, in order to increase the generalizability of our results, we conduct our sequence manipulation in a new decision context.

\section{Method}

Participants. Eighty-nine adults with American IP addresses $(57.3 \%$ female, mean age $=36.9$ years $)$ were recruited for this study from a large online subject pool. Participants were paid a fixed sum $(\$ 0.40)$ plus the possibility of earning up to $\$ 1$ extra depending on their performance in a bonus task.

Design and Procedure. The study was described to participants as a "Cartoon Caption Study." Participants were told they would see three cartoons and were instructed to select a caption for each cartoon from the available choice set of captions. Each caption was numbered sequentially, and the choice-set size was prominently displayed on the button interface (a graphic depiction of the task is shown in fig. 6). Participants could advance through the choice set of possible captions, one at a time, using "next" and "previous" buttons. When participants decided on a caption for 


\section{FIGURE 6}

\section{EXPERIMENT 5: REPRESENTATION OF USER DISPLAY FOR CARTOON TASK}

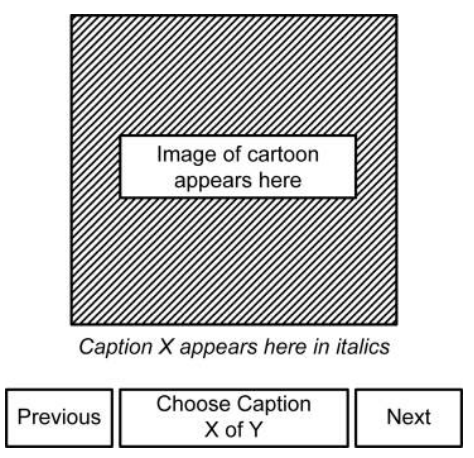

NoTE. $-Y$ is the total number of available captions in the choice set, and $X$ is the caption that is currently being displayed. Clicking Next would advance to caption $\mathrm{X}+1$.

the cartoon, they would select the caption using a "choose this caption" button. The program then proceeded to the next cartoon. The cartoons used as stimuli were borrowed from a major publication known to feature simple, singlepane cartoons. For each of the three cartoons, we randomly selected the choice set from a list of 500 user-generated captions.

We randomly assigned participants to either an increasing or decreasing condition. In the increasing condition, participants first encountered a cartoon with a choice set of 50 possible captions, then a cartoon with a choice set of 150 possible captions, and finally a cartoon with a choice set of 250 possible captions. Participants in the decreasing condition encountered the reverse sequence (i.e., 250 captions, 150 captions, and 50 captions). We also counterbalanced the order of the cartoons (i.e., half the participants saw the three cartoons in one order, and the other half saw them in the reverse order). This yielded a 2 (sequence: increasing vs. decreasing) $\times 2$ (cartoon order: normal vs. reversed) $\times 3$ (choice-set size: 50, 150, 250) mixed design with condition and cartoon order as between-participants factors and choiceset size as a within-participant factor.

After completing the cartoon caption task, all participants were then given an additional, bonus task, which was structured so that persistence would be rewarded with a higher bonus payment. Participants were told that they would "draw" a random bonus payment (generated from a quasi-normal distribution bounded at $\$ 0.01$ and $\$ 1.00$ ). If participants were satisfied with their draw, they could accept it. However, participants could continue drawing new bonuses as long as they desired - the final bonus payment would be the largest overall bonus draw (this value was clearly displayed on the screen). The only penalty for drawing was time: each draw took 5 seconds to materialize. The exact instructions to participants are provided in appendix B.

We predicted that participants in the increasing condition would adopt a maximizing mind-set and thus would persist longer in the bonus task by taking more bonus draws than their counterparts in the decreasing condition. This bonus task provides a strong test of our maximizing-mind-set hypothesis because it is in a completely different domain and is also clearly incentive compatible (more persistence will statistically lead to greater payment). Note that it is possible to consider this bonus task a search task in which participants are asked to search for an acceptable bonus draw, with the possibility of reinspecting previously drawn bonuses.

\section{Results}

Caption Search Depth. We operationalized search depth as the total number of captions viewed for each cartoon (i.e., how deeply the participant advanced into the caption choice set). We submitted search depth to a $2 \times 2 \times 3$ repeatedmeasure ANOVA with sequence (increasing vs. decreasing), cartoon order, and choice-set size as independent variables. Consistent with the previous studies, we found a main effect of choice-set size $(F(2,170)=13.22, p<.001)$. Participants in both conditions searched more captions in the larger choice sets. More importantly, we replicated the effect of sequence on depth of search: participants in the increasing condition searched more captions $\left(M_{\text {total }}=154\right.$ captions $)$ than those in the decreasing condition $\left(M_{\text {total }}=88\right.$ captions; $F(1,85)=8.57, p<.01)$. Planned contrasts revealed significant differences between conditions in depth of search for each of the three set sizes (all $F>5$, all $p<.03$ ). We find the same pattern of results when we analyze total search time as the dependent measure $\left(M_{\text {inc. }}=458\right.$ seconds, $M_{\text {dec. }}$. $=268$ seconds; $F(1,85)=8.93, p<.01)$. Although we unexpectedly found a main effect of the counterbalancing factor on search depth $(F(1,85)=5.87, p=.02)$, the nonsignificant interaction between the sequence manipulation and counterbalancing factor $(F(1,85)=.39, p>.5)$ indicates that counterbalancing did not influence the relationship between the sequence manipulation and depth of search. The results are illustrated in figure 7.

Persistence at Bonus Task. If the relative tendency to maximize induced by our manipulation carries over to subsequent tasks, we should also observe participants in the increasing condition striving for higher payouts in the bonus task. This should be reflected in their willingness to incur a higher cost (in time) for additional bonus draws (5 seconds per draw). Because draws carry no risk of lower bonus payments, a (relative) maximizer should continue drawing longer than a (relative) satisficer, who should be more likely to settle for an earlier draw. Consistent with our hypothesis, participants in the increasing condition drew more times $(M$ $=52.3)$ than their counterparts in the decreasing condition $(M=27.8 ; F(1,87)=3.89, p=.05)$. In other words, participants in the increasing condition were willing to accept the cost of striving for a higher payout when their 
FIGURE 7

EXPERIMENT 5: DEPTH OF SEARCH AND BONUS TASK PERSISTENCE
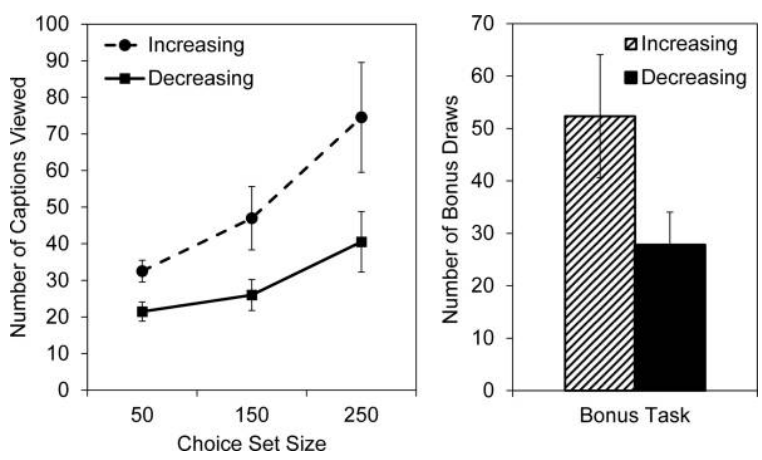

decreasing condition counterparts settled for their current payout. To their chagrin, participants in the increasing condition did not actually receive a significantly higher bonus payout $\left(M_{\text {inc. }}=\$ 0.78, M_{\text {dec. }}=\$ 0.77 ; F(1,87)=.22, p=\right.$ $.83)$. This is not surprising, however, due to the nature of distribution of the bonus draws: mathematically, the expected value of drawing 52 times rather than 28 times was only 3 cents.

\section{Discussion}

The results of experiment 5 provide further evidence that our manipulation evoked a maximizing mind-set in our participants. We show that the tendency to maximize carries over from the initial task to a task in a completely different domain. Further, like in experiment 4, this result is inconsistent with a resource-depletion account: participants in the increasing condition expended more effort on search in the sequence task and then continued expending more effort by persisting at the bonus task.

\section{GENERAL DISCUSSION}

The current research contributes to a growing literature at the intersection of consumer search and choice (e.g., Häubl et al. 2010; Kim, Albuquerque, and Bronnenberg 2010). In this article, we test the hypothesis that ordering sequential decisions by set size affects consumers' search behavior by leading to a stronger tendency to maximize or satisfice. Further, we show that this tendency can carry over to subsequent tasks. Across five experiments and multiple operationalizations of search depth (sampling count, decision time, and information acquisition), we find that ordering by increasing (vs. decreasing) choice-set sizes leads to greater search. In experiment 1 , we asked participants to configure a 10-track music $\mathrm{CD}$, one track at a time, each track from a choice set of a different size. We find that a sequence of increasing choice-set sizes leads to more sampling than a decreasing sequence. In experiment 2 , we generalize these results to search goods and use a different measure of search depth (information acquisition). In experiment 3, we cast doubt on the alternative explanation that our sequence effect is due to participants' erroneous expectations about the set sizes that they may encounter in the experiment. More importantly, in this experiment, we establish the link between maximizing versus satisficing mind-sets and people's search behavior under different decision sequences. Specifically, people in an increasing set-size condition both searched more and reported engaging in a strategy that consisted of locating the best option available in the choice set. When compared to a baseline condition of equal-sized choice sets, this difference in search appears to be driven by greater search in the increasing set-size condition. This suggests that an increasing sequence of choice-set sizes is effective at evoking a maximizing mind-set in participants, who otherwise would be more inclined to satisfice. In addition, in experiment 3, we find that the downstream effects on our participants' satisfaction are consistent with research showing that chronic maximizers search more but are less satisfied with their choices. In experiment 4 , we replicate our results using a much shorter sequence of decisions and show that the maximizing tendency triggered by the decision sequence carries over to a subsequent, unexpected task in the same domain. Finally, in experiment 5, we bolster the mind-set account by showing that the tendency to maximize can carry over to a subsequent task in a completely different domain as well.

\section{Is One Choice Set Enough?}

A lingering question is the extent to which the first choice in the decision sequence influences the adopted mind-set. It is possible, for instance, that the mind-set adopted is reinforced by the gradual pattern of increasing or decreasing set size. In other words, is a small initial choice set sufficient to initiate a maximizing mind-set, or is a gradual pattern of increases also critical? We conducted two additional followup studies to examine this question. In both studies, we compared a sequence with gradually increasing choice-set sizes to one with a more abrupt increase in choice-set size (e.g., gradual-increase choice-set sizes: 50, 100, 150, and 200 vs. abrupt-increase choice-set sizes: 50, 50, 200, and 200). In both studies, we observed a decrease in total search in the abrupt-increase condition, although this difference only reached significance in one of the two studies. These results suggest that the first choice set in the sequence is the most influential in determining the mind-set that the consumer adopts. The results also indicate that the pattern of increase may play a role in maintaining the mind-set: abrupt increases seem to reduce the total level of search. This possibility is supported by research on learning, person perception, and confirmatory processing, which demonstrate that people are unlikely to update their beliefs unless they encounter a salient failure of their initial hypothesis (Hastie and Kumar 1979; Hoch 1984; Hoch and Ha 1986; Srull, Lichtenstein, and Rothbart 1985). In this case, a large jump 
in choice-set size may cause the consumer to override her mind-set and reconsider her search strategy.

\section{A Percent-to-Search Heuristic?}

We contend that the differences in search depth that we observe in our experiments are driven by the formation of different mind-sets. However, another possibility is that participants develop some type of rule of thumb to guide their search behavior. In particular, participants might adopt a "percent-to-search" heuristic based on a target percentage of the choice set they believe that they should sample (e.g., "always examine $80 \%$ of the options"). Such a heuristic would give rise to a difference in total search levels similar to those we observe if participants in the increasing condition were adopting a high percent-to-search target based on their initial encounter with a small choice set, and participants in the decreasing condition were adopting a low percent-to-search target based on their initial encounter with a large choice set. Our data allow us to rule out this heuristic in its strictest sense. First, across all the experiments, we observe large differences in percent searched between the biggest and smallest sets (e.g., for experiment 1, this is illustrated in the bottom panel of fig. 2). Second, the bonus task in experiment 5 casts doubt on the viability of a strict percent-to-search heuristic, as such a heuristic could not guide a participant's search behavior in the bonus task in which search is limitless (participants could in principle draw bonuses indefinitely).

However, a looser interpretation of a percent-to-search heuristic is more difficult to rule out. Under such an interpretation, participants would adopt a target for search based on a rough sense of the set size (e.g., "this is a small set" or "this is a big set") and what proportion of the set would be reasonable to search (e.g., "I'll search a lot of the options" or "I'll search a few of the options"). Although this target proportion might shift as participants encountered new set sizes, the initial target proportion could serve as an anchor. Like different mind-sets, such a process would also lead to different total levels of search between the increasing and decreasing conditions. The key difference between this account and a mind-set account is that the percent-to-search heuristic entails a process-based goal (i.e., to search a certain amount), while a mind-set account entails an outcome-based goal (i.e., to choose a sufficiently satisfying item). In this regard, experiment 3 offers evidence favoring mind-sets because we observe differences both in reported maximizing tendency and in downstream satisfaction. The latter, in particular, is difficult to explain with a percent-to-search heuristic but is consistent with prior research on maximization.

\section{Bounded Adaptivity}

Our findings raise several theoretical and practical implications. First, past research using one-shot decisions offers compelling evidence that consumers adapt their decision strategies according to the requirements of the choice task (Bettman, Luce, and Payne 1998; Payne et al. 1993). Our results indicate that people are actually "sticky adapters" whose strategies are adapted to new contexts - such as the initial choice set-but persist to a significant degree even in the face of changes in the decision environment. The adaptivity in our experiments is evident in the changes in percentage searched between the small and large choice sets. The stickiness in our experiments is evident in the difference in cumulative search between participants in the increasing and decreasing conditions. Second, our research indicates that the effect of choice-set size on motivation may be contingent on consumers' previous experience. In particular, past research shows that consumers faced with a large number of options are less motivated to make a purchase (Iyengar and Lepper 2000). Yet, participants in our experiments who were initially exposed to small choice sets and then were exposed to larger choice sets appeared to "keep up" and remained more motivated, as indicated by their more extensive search relative to those who saw the reverse sequence. Third, we provide evidence suggesting that maximizing - typically studied as a chronic trait (e.g., Schwartz et al. 2002)_can be also be triggered by the decision situation. Finally, on the practical front, our experiments suggest a simple manipulation for managers to increase customer search and, perhaps, engagement: simply order decisions according to ascending choice-set size. At the same time, we caution that this deeper search could potentially lead to decreased postchoice satisfaction.

Our results complement recent research by Levav et al. (2010) on the effect of set-size order on automobile customization decisions. In particular, their research manipulates the sequence in which attribute decisions are made and shows that customers are more likely to accept the default attribute level in small choice sets when they follow rather than precede large choice sets. In other words, in decreasing sequences, auto buyers were more likely to accept the default attribute level as they proceeded through their customization decisions. One potential interpretation of accepting the default option in the Levav et al. (2010) study is that the default represents the "good enough" option. Thus, taking the default may be a behavioral by-product of a satisficing mindset. This is congruent with the results we obtain in our decreasing sequence conditions.

Note, however, that there are also several important differences between our work and Levav et al.'s (2010) work. First, our article is explicitly concerned with depth of search, whereas Levav et al. (2010) did not measure the number of options that customers viewed before making their decision. Second, the Levav et al. (2010) article is concerned with testing the validity of a basic assumption that underlies differentiated product models (that the utility from a product is the summation of the utilities from its attributes), whereas here we are concerned with a psychological factor that explains different search behaviors in sequential choices. To this end, we measure participants' reported levels of maximization and show that the consequences of the order manipulation extend to subsequent tasks. 


\section{Future Directions}

Our findings also suggest several directions for future research. First, although we focused this inquiry on the effect of ordering by choice-set size, it is easy to conjure other types of psychologically meaningful attribute orderings that might affect consumer search and decision making, such as attribute importance, price, or trade-off difficulty. Another possibility is to study the interplay between choice-set ordering and other important psychological variables such as attention, expectations, satiation, and regret. A second direction is to study the limits of the effect of mind-sets on search. Although the hallmark of mind-sets is that they carry over to subsequent tasks, induced decision mind-sets-like those in our studies-must be reset at some point. When does this reset occur? Research on evaluation of hedonic experiences shows that when there is a sensory discontinuity, people segment an experience into parts and become less sensitive to its overall pattern (Ariely and Zauberman $2000,2003)$. One can imagine that a salient interruption like a pop-up ad or a change of web page styles in the midst of an online product configuration process may reset-or partially reset-the mind-set evoked by previous decisions. The additional experiments reported in the general discussion begin to explore this issue by examining the consequences of abrupt changes in choice-set size. Third, there may be an interaction between the order of attributes and the order of options within each attribute. For instance, will consumers who have adopted a maximizing mind-set make better decisions when offered an ordered option set rather than a random set? Research has found that search depth is reduced when options within an attribute are ordered by descending quality, which may lead to a lower overall evaluation of the set than when the set is ordered by ascending quality (Diehl and Zauberman 2005). It is possible that the ordering of attribute decisions moderates search depth and hence the overall evaluation of the options within each attribute. Finally, future research may examine whether decision sequence interacts with sales message content. For instance, people in a maximizing mind-set might find certain sales messages, such as those that stress product comparisons, more appealing than those in a satisficing mind-set.

\section{Conclusion}

Sequential decisions are ubiquitous in the marketplace. Absent an understanding of the effect of decision order, firms typically create customer interfaces using an engineer's perspective about how decisions should be sequenced: a series of attribute decisions that begins with the central component and proceeds to the peripheral components (e.g., a car configuration begins with the engine). This article highlights opportunities for firms to influence their customers' behavior by ordering a decision sequence based on psychologically meaningful variables.

\section{APPENDIX A}

\section{EXPERIMENT 3: SELF-REPORTED MAXIMIZING SEARCH GOALS}

Please indicate your degree of agreement with the following statements $(-3=\mathrm{I}$ do not agree with the statement at all, $+3=$ I agree with the statement very much):

1. Even if I found a song that I was relatively satisfied with, I still listened to the other available songs before deciding.

2. I tried to find the best song for each track.

3. I had in mind some ideal song(s) for the CD, and I tried to look for songs similar to my ideal song(s).

4. I made my decision for each track as soon as I found a song that was good enough. (reverse coded)

\section{APPENDIX B \\ EXPERIMENT 5: INSTRUCTIONS FOR BONUS TASK}

As a thank you for participating in this study, we are going to give you a bonus payment. Your bonus payment will be calculated using a game. The rules are simple. You will "draw" a random value between $\$ 0.01$ and $\$ 1.00$. If you are satisfied with this draw, you can click the Accept Bonus button. However, if you want a bigger bonus, you keep drawing new bonuses. Your bonus will be your highest draw. In other words, you cannot lose money by drawing -you can only make your bonus bigger!!!

\section{REFERENCES}

Amir, On, and Jonathan Levav (2008), "Choice Construction versus Preference Construction: The Instability of Preferences Learned in Context," Journal of Marketing Research, 45 (2), 145-58.

$\rightarrow$ Ariely, Dan, and Gal Zauberman (2000), "On the Making of an Experience: The Effects of Breaking and Combining Experiences on Their Overall Evaluation," Journal of Behavioral Decision Making, 13 (2), 219-32.

$\rightarrow$ _ _ (2003), "Differential Partitioning of Extended Experiences," Organizational Behavior and Human Decision Processes, 91 (July), 128-39.

$\rightarrow$ Asch, Solomon E. (1946), "Forming Impressions of Personality," Journal of Abnormal and Social Psychology, 41 (3), 258-90.

$\rightarrow$ Baumeister, Roy, Ellen Bratslavsky, Mark Muraven, and Dianne M. Tice (1998), "Ego Depletion: Is the Active Self a Limited Resource?" Journal of Personality and Social Psychology, 74 (5), 1252-65.

Bem, Daryl J. (1972), "Self-Perception Theory," in Advances in Experimental Social Psychology, Vol. 6, ed. Leonard Berkowitz, New York: Academic Press, 1-62.

$\rightarrow$ Bettman, James R., Mary Frances Luce, and John W. Payne (1998), "Constructive Consumer Choice Processes," Journal of Consumer Research, 25 (December), 187-217. 
$\rightarrow$ Broder, Arndt, and Stefanie Schiffer (2006), “Adaptive Flexibility and Maladaptive Routines in Selecting Fast and Frugal Decision Strategies," Journal of Experimental Psychology: Learning, Memory, and Cognition, 32 (4), 904-18.

$\rightarrow$ Chernev, Alexander (2003), "When More Is Less and Less Is More: The Role of Ideal Point Availability and Assortment in Consumer Choice," Journal of Consumer Research, 30 (2), 170-83.

Dellaert, Benedict, G. C., and Gerald Häubl (2012), "Searching in Choice Mode: Consumer Decision Processes in Product Search with Recommendations," Journal of Marketing Research, forthcoming.

Dellaert, Benedict, G. C., and Stefan Stremersch (2005), "Marketing Mass-Customized Products: Striking a Balance between Utility and Complexity," Journal of Marketing Research, 42 (2), 219-27.

$\rightarrow$ Dhar, Ravi, Stephen M. Nowlis, and Steven J. Sherman (2000), "Trying Hard or Hardly Trying: An Analysis of Context Effects in Choice," Journal of Consumer Psychology, 9 (4), 189-200.

$\rightarrow$ Diehl, Kristin (2005), "When Two Rights Make a Wrong: Searching Too Much in Ordered Environments," Journal of Marketing Research, 42 (3), 313-22.

Diehl, Kristin, Laura J. Kornish, and John G. Lynch Jr. (2003), "Smart Agents: When Lower Search Costs for Quality Information Increase Price Sensitivity," Journal of Consumer Research, 30 (1), 56-71.

$\rightarrow$ Diehl, Kristin, and Gal Zauberman (2005), "Searching Ordered Sets: Evaluations from Sequences under Search," Journal of Consumer Research, 31 (4), 824-32.

$\rightarrow$ Goukens, Caroline, Siegfried DeWitte, and Luk Warlop (2009), "Me, Myself, and My Choices: The Influence of Private SelfAwareness on Choice," Journal of Marketing Research, 46 (5), 682-92.

$\rightarrow$ Griffin, Jill G., and Susan M. Broniarczyk (2010), "The Slippery Slope: The Impact of Feature Alignability on Search and Satisfaction," Journal of Marketing Research, 47 (April), 32334.

Hastie, Reid, and Purohit A. Kumar (1979), "Person Memory: Personality Traits as Organizing Principles in Memory for Behaviors," Journal of Personality and Social Psychology, 37 (1), 25-38.

$\rightarrow$ Häubl, Gerald, Benedict G. C. Dellaert, and Bas Donkers (2010), "Tunnel Vision: Local Behavioral Influences on Consumer Decisions in Product Search," Marketing Science, 29 (3), 438-55.

$\rightarrow$ Häubl, Gerald, and Kyle B. Murray (2003), "Preference Control and Persistence in Digital Marketplaces: The Role of Electronic Recommendation Agents," Journal of Consumer Psychology, 13 (1-2), 75-91.

Hoch, Stephen J. (1984), "Hypothesis Testing and Consumer Behavior: If It Works, Don't Mess with It," in Advances in Consumer Research, Vol. 11, ed. Thomas C. Kinnear, Ann Arbor, MI: Association for Consumer Research, 478-83.

$\rightarrow$ Hoch, Stephen J., and Young-Won Ha (1986), "Consumer Learning: Advertising and the Ambiguity of Product Experience," Journal of Consumer Research, 13 (2), 221-33.

$\rightarrow$ Iyengar, Sheena S., and Emir Kamenica (2010), "Choice Proliferation, Simplicity Seeking, and Asset Allocation," Journal of Public Economics, 94 (7-8), 530-39.

$\rightarrow$ Iyengar, Sheena S., and Mark R. Lepper (2000), "When Choice Is Demotivating: Can One Desire Too Much of a Good Thing,"
Journal of Personality and Social Psychology, 79 (6), 9951006.

$\rightarrow$ Iyengar, Sheena S., Rachael E. Wells, and Barry Schwartz (2006), "Doing Better but Feeling Worse: Looking for the 'Best' Job Undermines Satisfaction," Psychological Science, 17 (2), $143-50$.

$\rightarrow$ Johnson, Eric J., John W. Payne, and James R. Bettman (1988), "Information Displays and Preference Reversals," Organizational Behavior and Human Decision Processes, 42 (1), $1-21$.

Keinan, Anat, and Ran Kivetz (2011), "Productivity Orientation and the Consumption of Collectable Experiences," Journal of Consumer Research, 37 (6), 935-50.

Kim, Jun B., Paulo Albuquerque, and Bart J. Bronnenberg (2010), "Online Demand under Limited Consumer Search," Marketing Science, 29 (6), 1001-23.

Levav, Jonathan, Mark Heitmann, Andreas Herrmann, and Sheena S. Iyengar (2010), "Order in Product Customization Decisions," Journal of Political Economy, 118 (2), 274-99.

Luchins, Abraham S. (1942), "Mechanization in Problem Solving," Psychological Monographs, 54 (6), 1-95.

$\rightarrow$ Meyer, Robert (1997), "The Effect of Set Composition on Stopping Behavior in a Finite Search among Assortments," Marketing Letters, 8 (1), 131-43.

$\rightarrow$ Payne, John W. (1976), "Task Complexity and Contingent Processing in Decision Making: An Information Search and Protocol Analysis," Organizational Behavior and Human Performance, 16 (August), 366-87.

Payne, John W., James R. Bettman, and Eric J. Johnson (1988), "Adaptive Strategy Selection in Decision Making," Journal of Experimental Psychology, 14 (3), 534-52.

___ (1993), The Adaptive Decision Maker, New York: Cambridge University Press.

$\rightarrow$ Preacher, Kristopher J., and Andrew F. Hayes (2004), "SPSS and SAS Procedures for Estimating Indirect Effects in Simple Mediation Models," Behavior Research Methods, Instruments, and Computers, 36 (4), 717-31.

$\rightarrow$ Schwartz, Barry (1982), "Reinforcement-Induced Behavioral Stereotypy: How Not to Teach People to Discover Rules," Journal of Experimental Psychology: General, 111 (1), 23-59.

Schwartz, Barry, Andrew Ward, John Monterosso, Sonya Lyubomirsky, Katherine White, and Darrin R. Lehman (2002), "Maximizing versus Satisficing: Happiness Is a Matter of Choice," Journal of Personality and Social Psychology, 83 (5), 1178-97.

$\rightarrow$ Shah, Avni M., and George Wolford (2007), "Buying Behavior as a Function of Parametric Variation of Number of Choices," Psychological Science, 18 (5), 369-70.

$\rightarrow$ Simon, Herbert A. (1955), "A Behavioral Model of Rational Choice," Quarterly Journal of Economics, 69 (1), 99-118.

$\rightarrow$ __ (1956), "Rational Choice and the Structure of the Environment," Psychological Review, 63 (2), 129-38.

Simonson, Itamar, and Amos Tversky (1992), "Choice in Context: Tradeoff Contrast and Extremeness Aversion," Journal of Marketing Research, 29 (August), 281-96.

Srull, Thomas K., Meryl Lichtenstein, and Myron Rothbart (1985), "Associative Storage and Retrieval Processes in Person Memory," Journal of Experimental Psychology: Learning, Memory, and Cognition, 11 (2), 316-45.

$\rightarrow$ Ülkümen, Gulden, Amitav Chakravarti, and Vicki G. Morwitz (2010), "Categories Create Mindsets: The Effect of Exposure 
to Broad versus Narrow Categorizations on Subsequent, Unrelated Decisions," Journal of Marketing Research, 47 (4), 659-71.

$\rightarrow$ Wyer, Robert S., Jr., and Jing Xu (2010), “The Role of Behavioral Mind-Sets in Goal-Directed Activity: Conceptual Underpinnings and Empirical Evidence," Journal of Consumer Psychology, 20 (2), 107-25. $\rightarrow$ Xu, Jing, and Robert S. Wyer Jr. (2007), "The Effect of MindSets on Consumer Decision Strategies," Journal of Consumer Research, 34 (December), 556-66.

$\rightarrow$ Zhao, Xinshu, John G. Lynch Jr., and Qimei Chen (2010), "Reconsidering Baron and Kenny: Myths and Truths about Mediation Analysis," Journal of Consumer Research, 37 (August), 197-206.

CORRECTION.-Since this article was published online on January 25, 2012, corrections have been made. Under "Experiment 3," "Results," in the "Mediation Analysis" section's second paragraph, " $t(82)=5.06, p=.03$ " was changed to " $F(1,82)$ $=5.06, p=.03$ "; under "Experiment 5," "Results," in the first paragraph of "Persistence at Bonus Task," " $t(87)=3.89$, $p=.05$ " was changed to " $F(1,87)=3.89, p=.05$," and in that same section " $t(87)=.05, p=.83$ " was changed to " $F(1,87)=.22, p=.83$." These changes were made in both the online and the print versions of the article. Corrected on August 27, 2012. 